\title{
UNIFORMITY AND WAVEFRONT CONTROL OF OPTICAL FILTERS
}

Michael Vergöhl ${ }^{1}$, Chris Britze ${ }^{1}$, Tobias Zickenrott ${ }^{1}$, Andreas Pflug ${ }^{1}$, Klaus Mann², Bernd Schäfer², Jennifer Ahrens², Volker Kirschner ${ }^{2}$

1) Fraunhofer IST, Braunschweig, Germany

2) Laser Laboratory Göttingen, Germany

ITT AO/1-8541/15/NL/PS

3) ESA-ESTEC, Noordwijk, The Netherlands

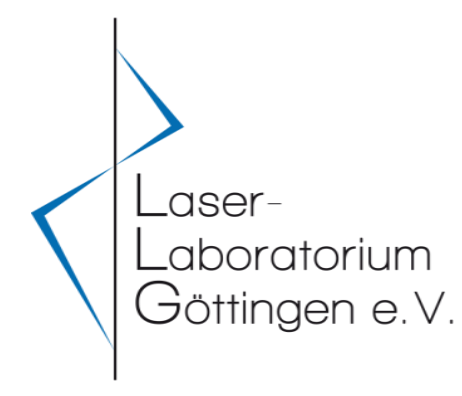




\section{Uniformity and wavefront control of optical filters}

Outline

1. Introduction - two different cases

2. Broadband beam splitter

- Coating design

- Measurement of spectral wavefront error

Results

3. Bandpass filter on a convex lens

- Design of masks by simulation

- Coating results

4. Summary, outlook 


\section{Introduction}

\section{Objectives of development}

- Uniformity control in low-loss optical coatings

- A) Broad band beam splitter

- Low wavefront error

- B) Bandpass filter on a lens

- Fixed bandpass position over lens area

- Useable e.g. for compact LIDAR
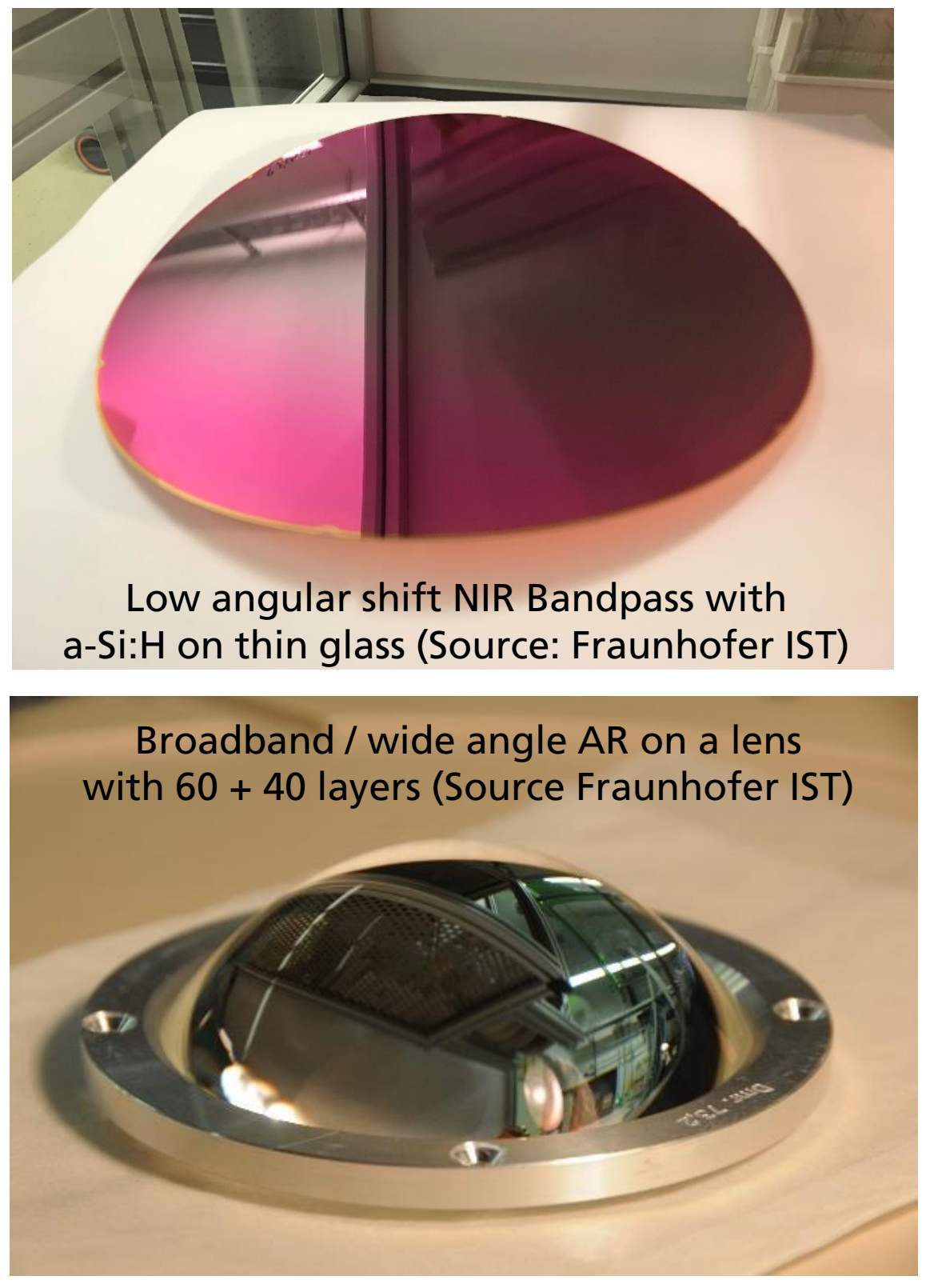


\section{Broadband beam splitter}

\section{Resonances in the wavefront error}

- Phase error calculation given by McLeod (figure right) and $P$. Baumeister

- Resonances in WFE observed on a beam splitter by Venancio et al. [1]

- Group delay is equivalent to wavefront error, the difference is the light speed in the media [2]

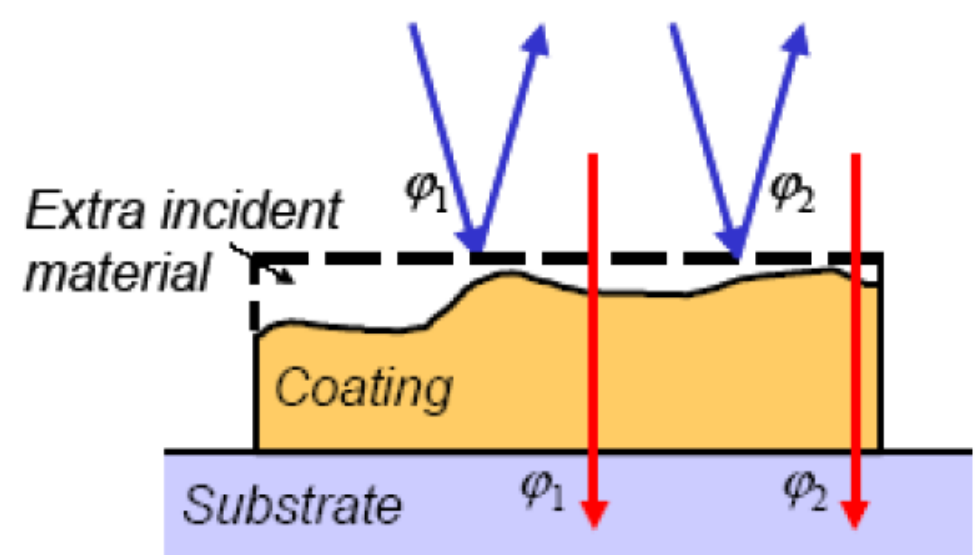

Angus McLeod, "Phase matters", 31 May 2005, SPIE Newsroom. DOI: $10.1117 / 2.5200506 .0008$

[1] Luis M. Gaspar Venancio et al., "Coating induced phase shift and impact on Euclid imaging performance", Proc. SPIE 9904-87 (2016), https://doi.org/10.1117/12.2232842

[2] M. Vergöhl, et al., Proc. SPIE 10691, Advances in Optical Thin Films VI, 1069118 (6 June 2018); 


\section{Broadband beam splitter}

Conditions

- Substrate $d=120 \mathrm{~mm}$ Thickness $12 \mathrm{~mm}$

- $\mathrm{AOI} 30^{\circ}$

- HR 400-900nm: $R_{a}>98 \%$

- HT 920-2300nm: $\mathrm{T}_{\mathrm{a}}>92 \%$

- WFE $<20$ nm r.m.s.

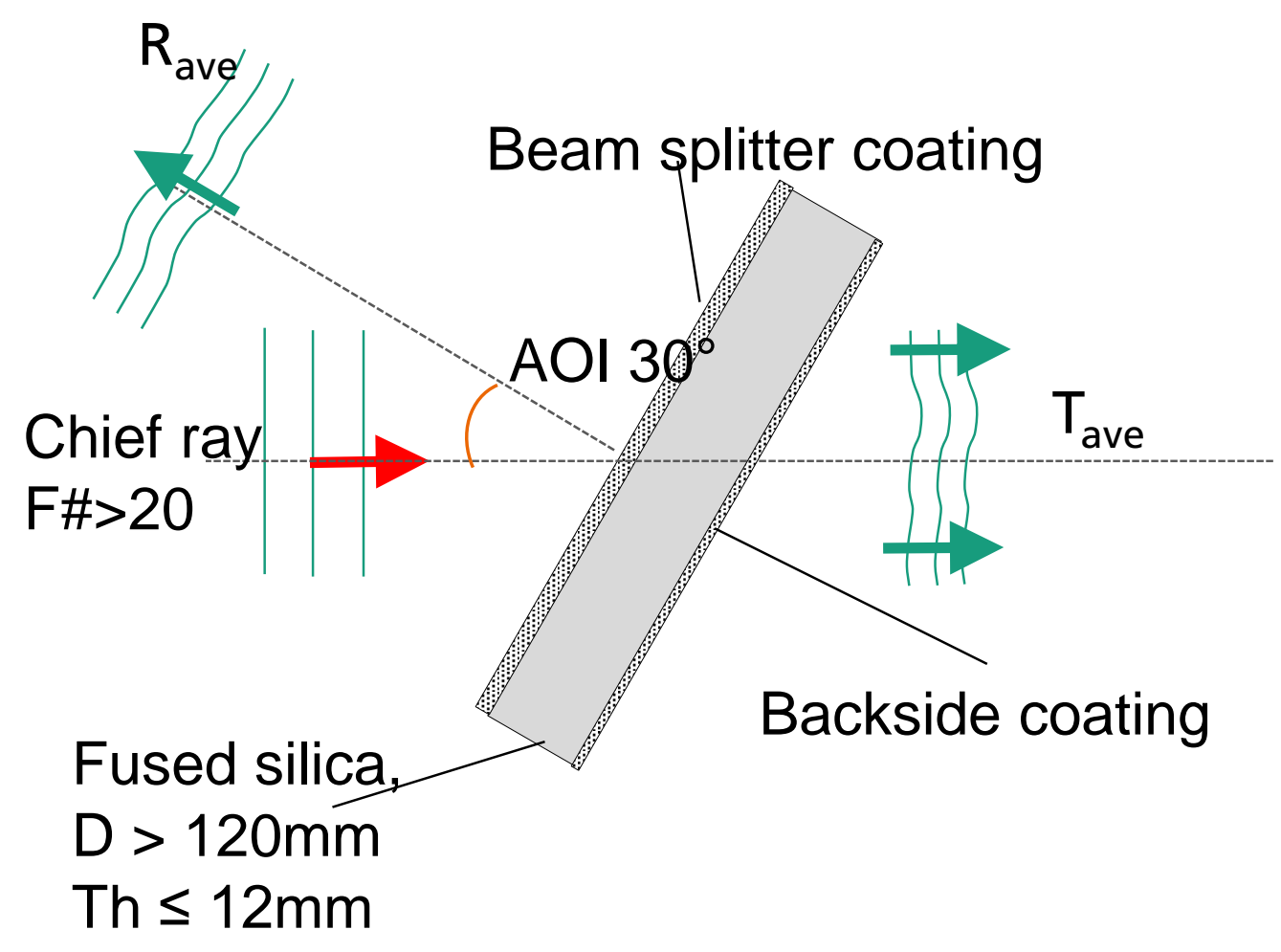




\section{Broadband beam splitter Wavefront measurement: Setup at LLG}

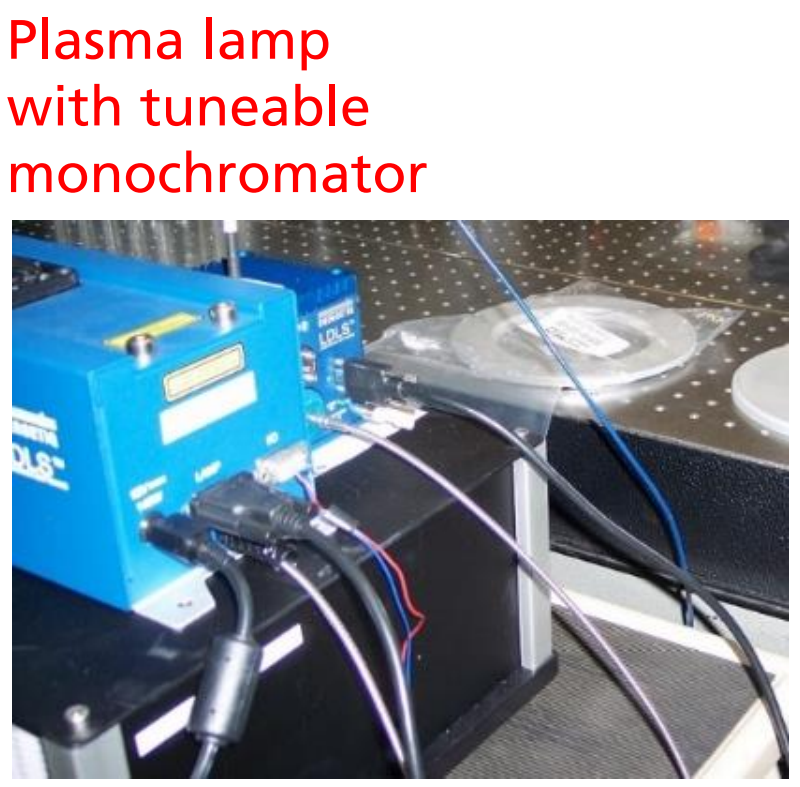

Hartmann-Shack wavefront sensor

K. Mann et al., "Spectrally resolved wavefront measurements on broad-band dielectric Coatings", proc. SPIE Laser Damage (2018), to be published

Sample / reference
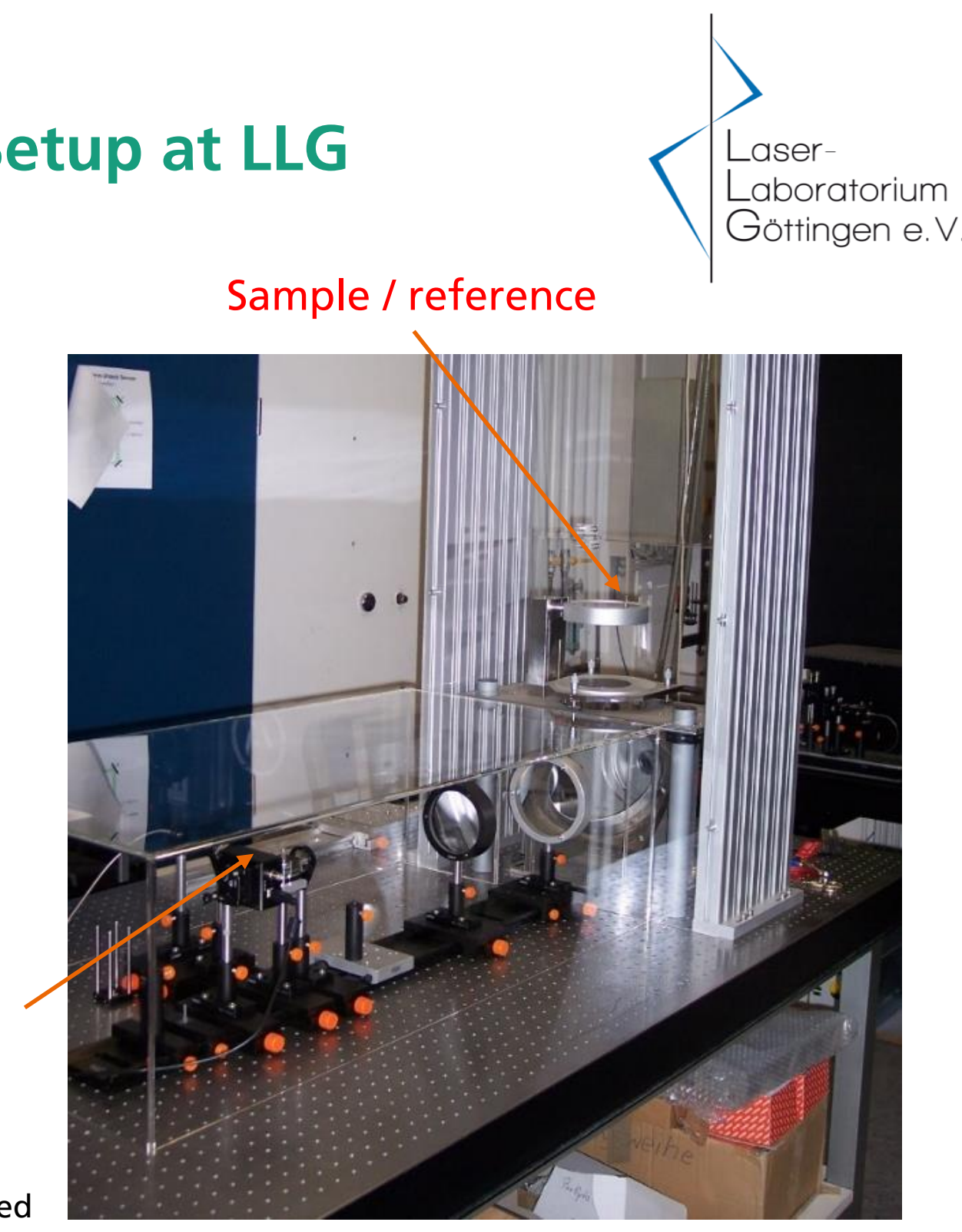


\section{Broadband beam splitter \\ Combination procedure to determine WFE spectra}

1) "Absolute" measurement

- Measure against a "FLAT" as reference

- Slow => Only a few wavelengths, used for referencing

- Sample holder features corrected by rotation of sample

- Bending by gravity corrected by uncoated identical substrate

- Substrate figure errors by measurement on uncoated substrate

- Bending by coating stress included

2) "Relative" measurement: Change wavelength only

- Sample itself is reference

- Fast, but needs a reference

Theory: Effect of non-uniform "air layer" included

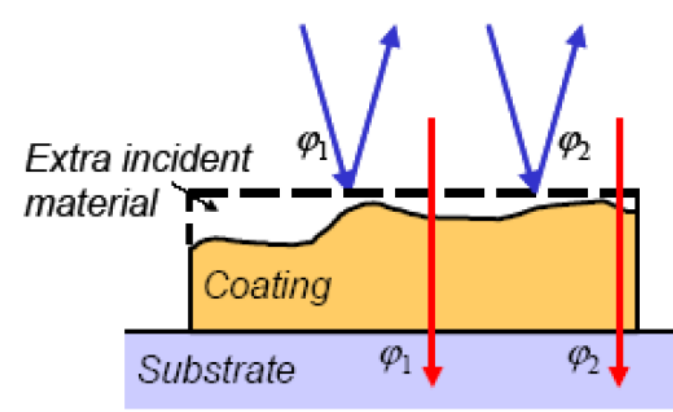




\section{Broadband beam splitter Relative WFE measurement}

- Earlier test coating

- about $0.5 \%$ non-uniformity $(+/-0.25 \%)$

- 112 layers, 9500nm thickness (only one side coated)

- Does not meet overall requirements for $R$ and $T$ around edge $=>$ improved design

- WFE: Agreement with theory
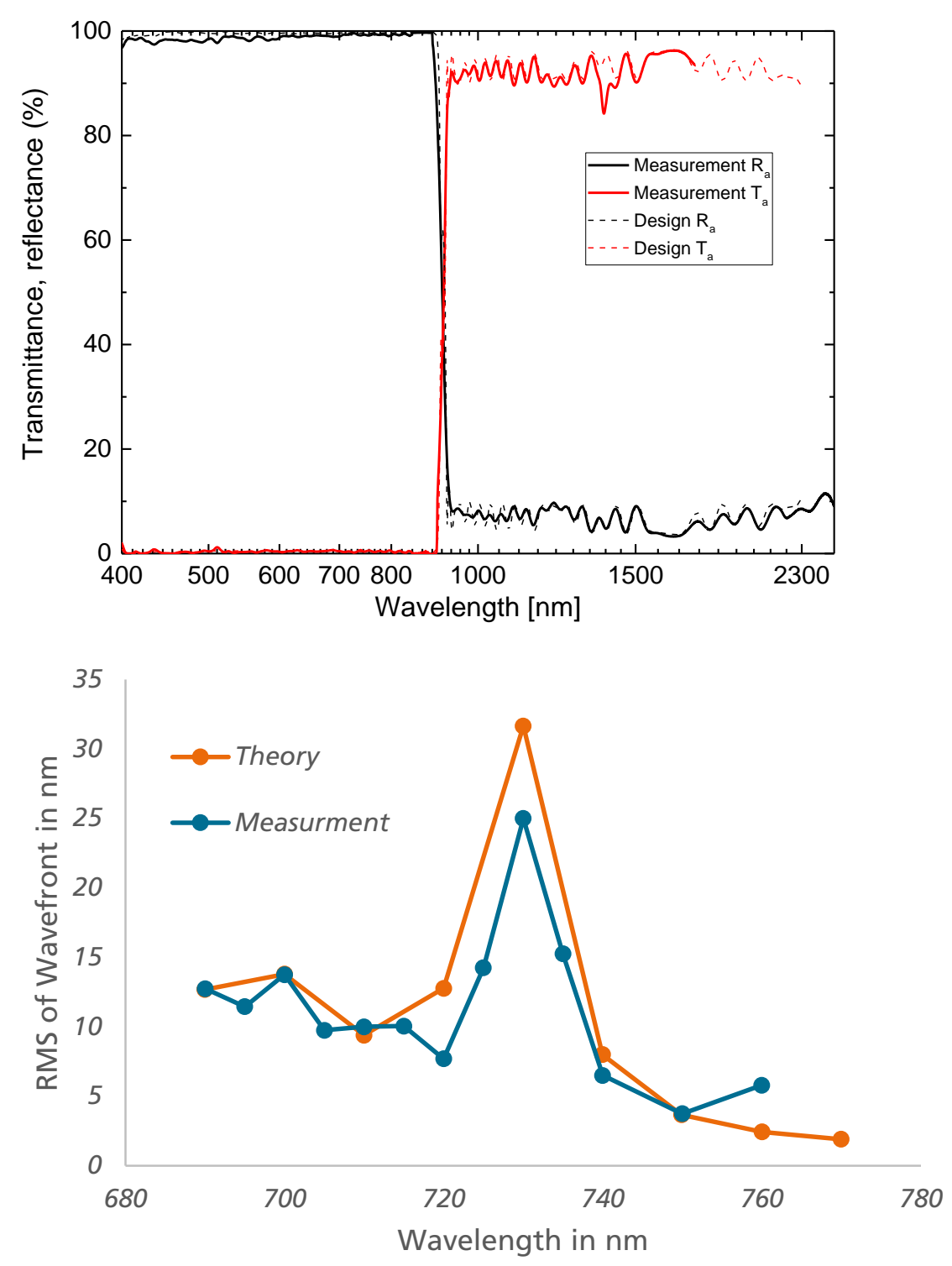


\section{Broadband beam splitter Manufacturing process (EOSS ${ }^{\circledR}$ system)}

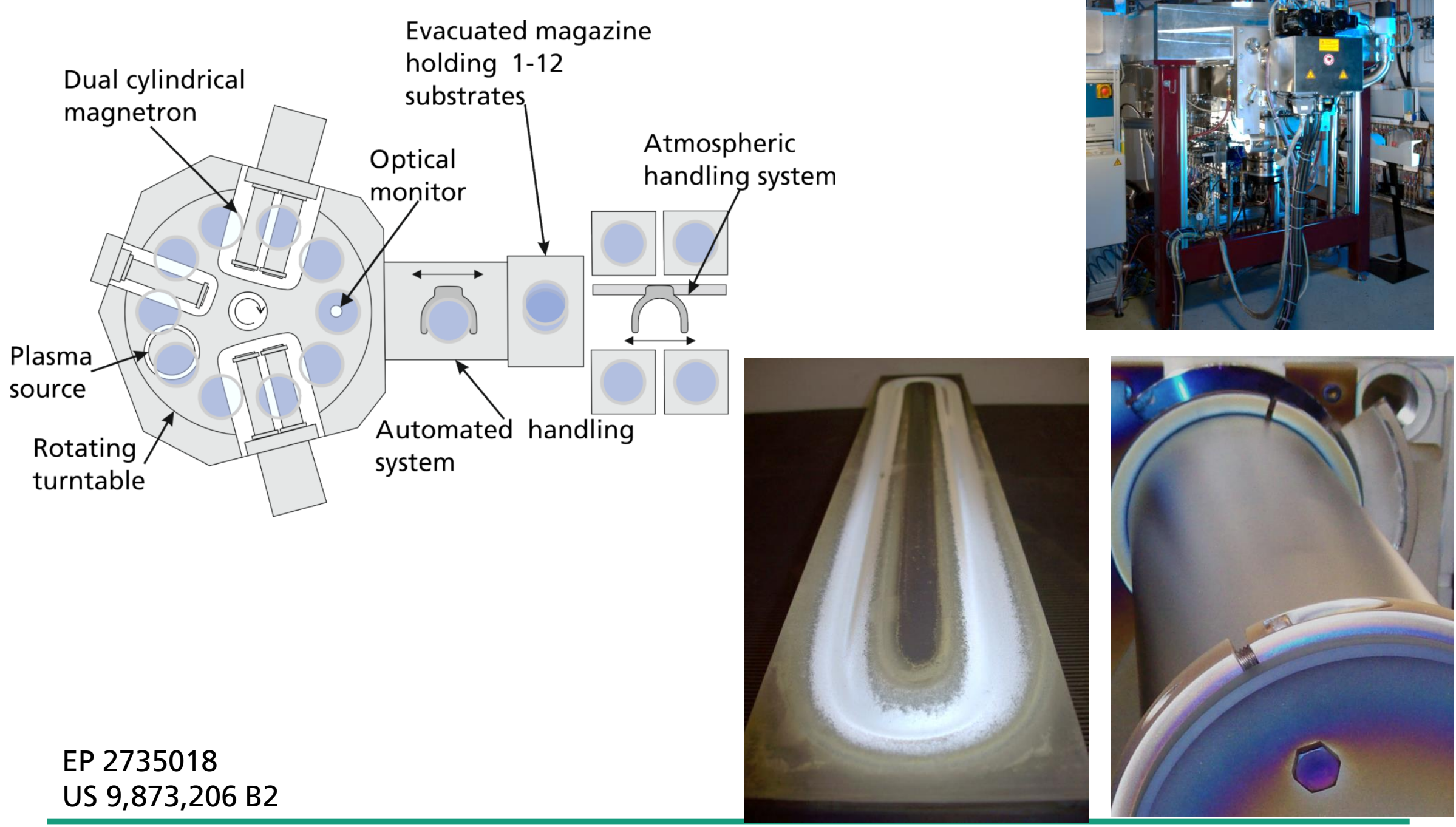

Evacuated magazine holding 1-12

ubstrates 


\section{Broadband beam splitter Final coating with improved design}

Ex-situ measurement (Perkin Elmer), $30^{\circ} \mathrm{AOI}$

- Design: 150 layers $\mathrm{SiO}_{2}+\mathrm{Ta}_{2} \mathrm{O}_{5}$

- 14,4 $\mu \mathrm{m}$ thickness

- WFE optimized

- Identical coating on both sides

- No post anneal

- Perkin Elmer measurement: Not suitable (beam divergence)

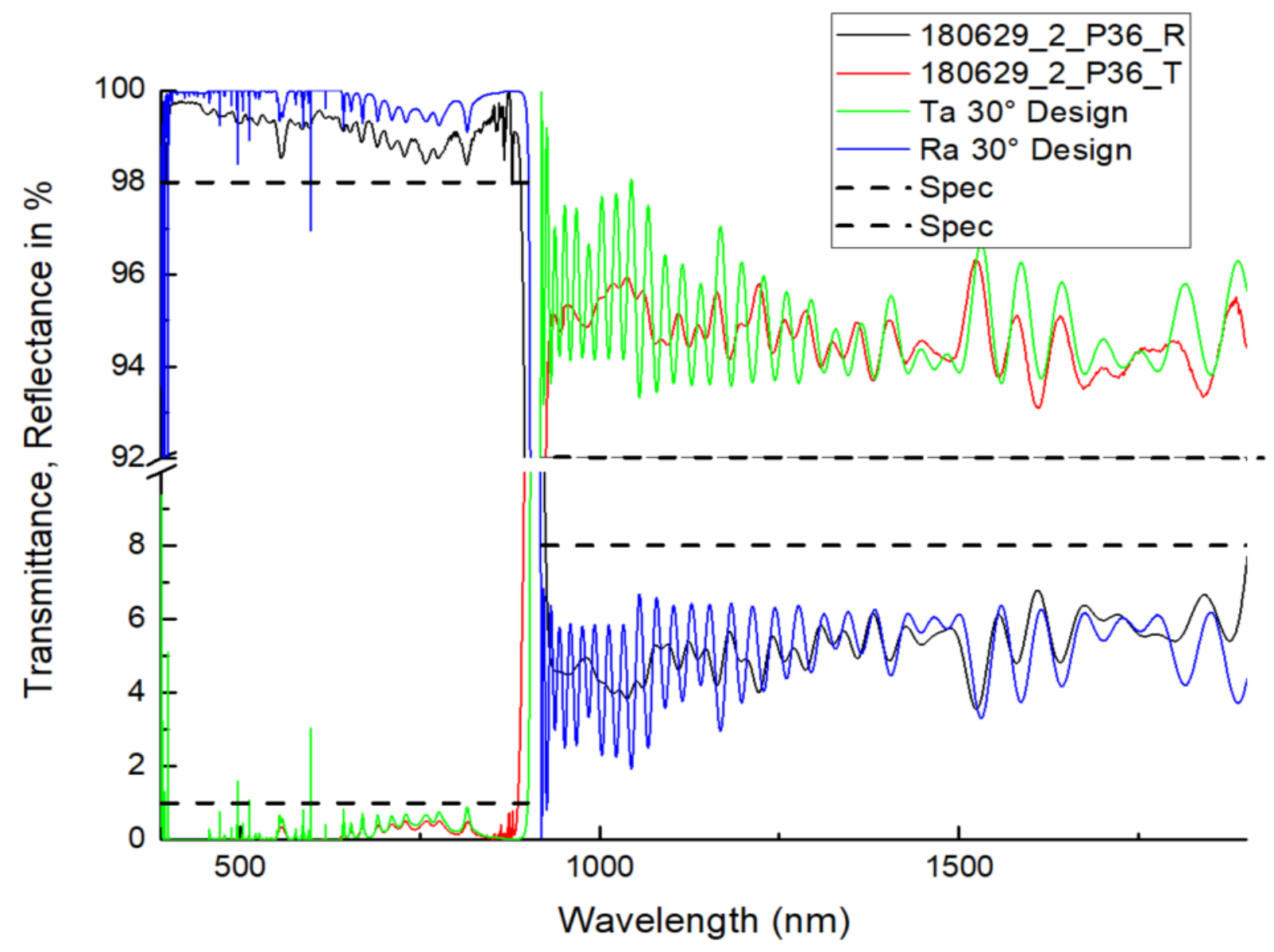




\section{Broadband beam splitter \\ Transmittance around the band edge}

- Use of self made spectrometer (divergence $<0.25^{\circ}$ )

- Deviation: 0,5nm $(0,06 \%)$ was shifted by tooling

- Non-uniformity: $\pm 0.125 \%$ over $140 \mathrm{~mm}$

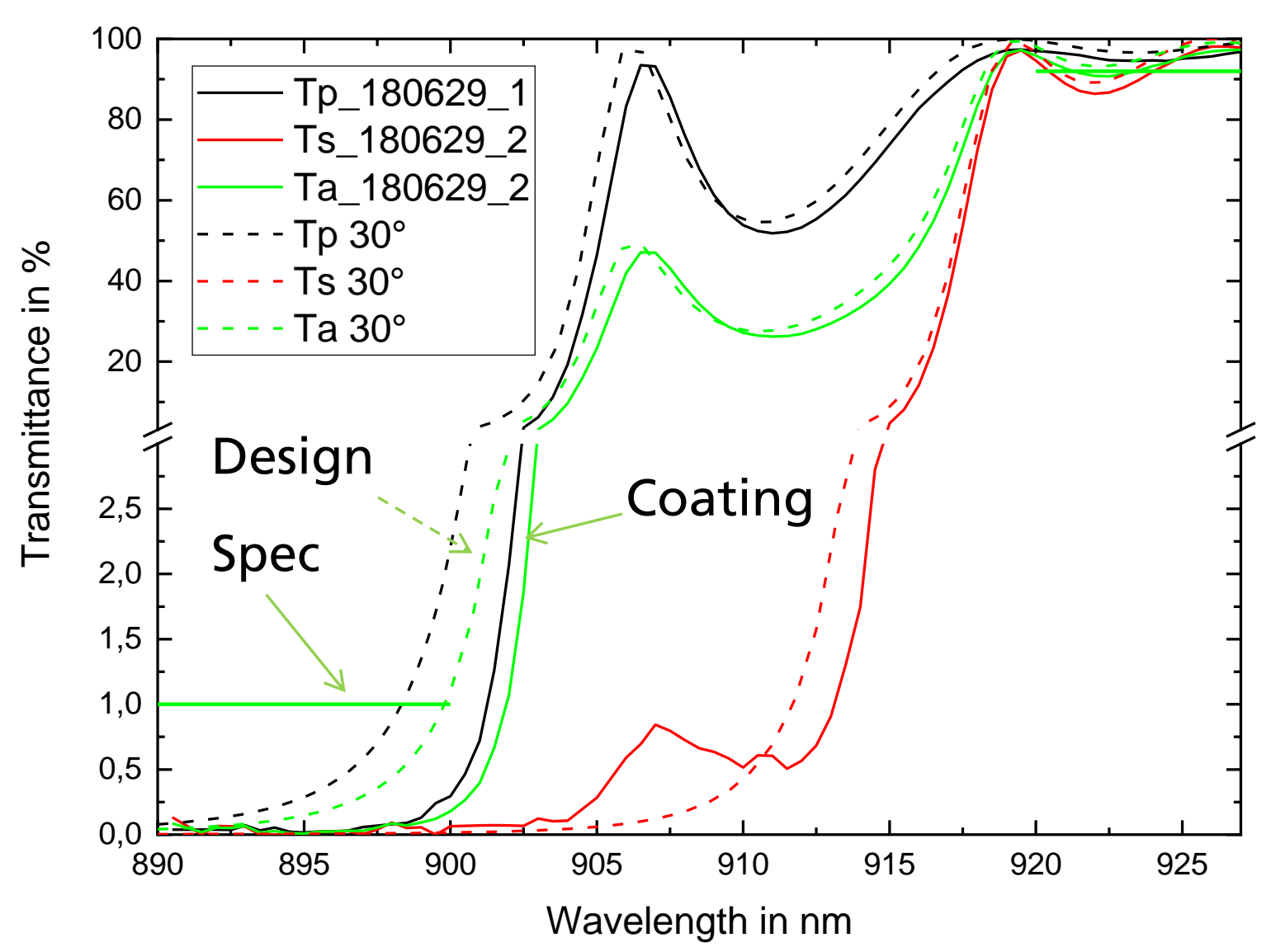




\section{Broadband beam splitter}

\section{In-situ MOCCA ${ }^{\circ}$ spectra (5 test glasses)}
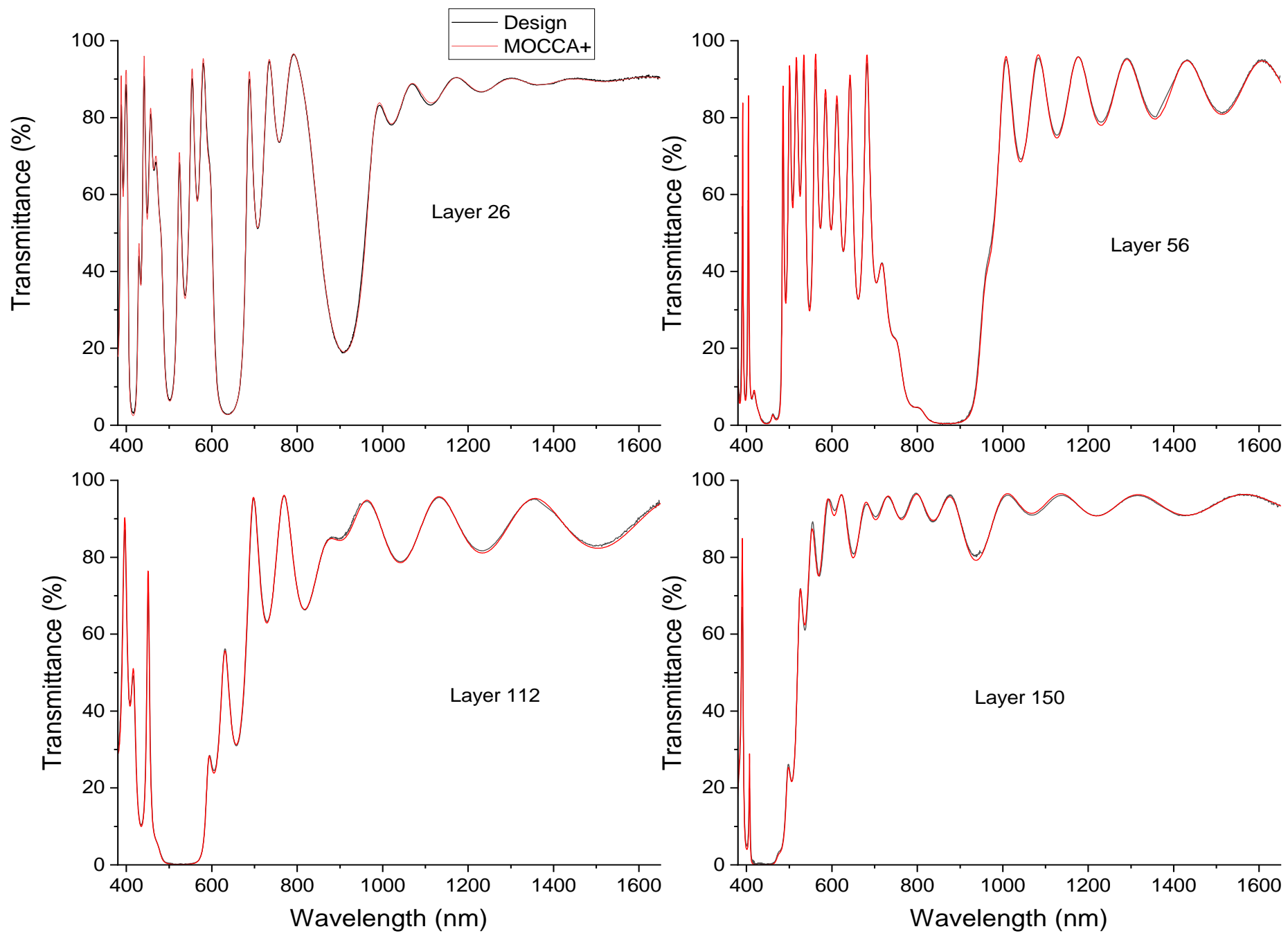


\section{Broadband beam splitter Spectral wavefront error result of final coating}

- Green: absolute WFE

- Red: Relative WFE

- Black: Theory

no bending observed within accuracy

- Future: Noise and chromatic offset reduction
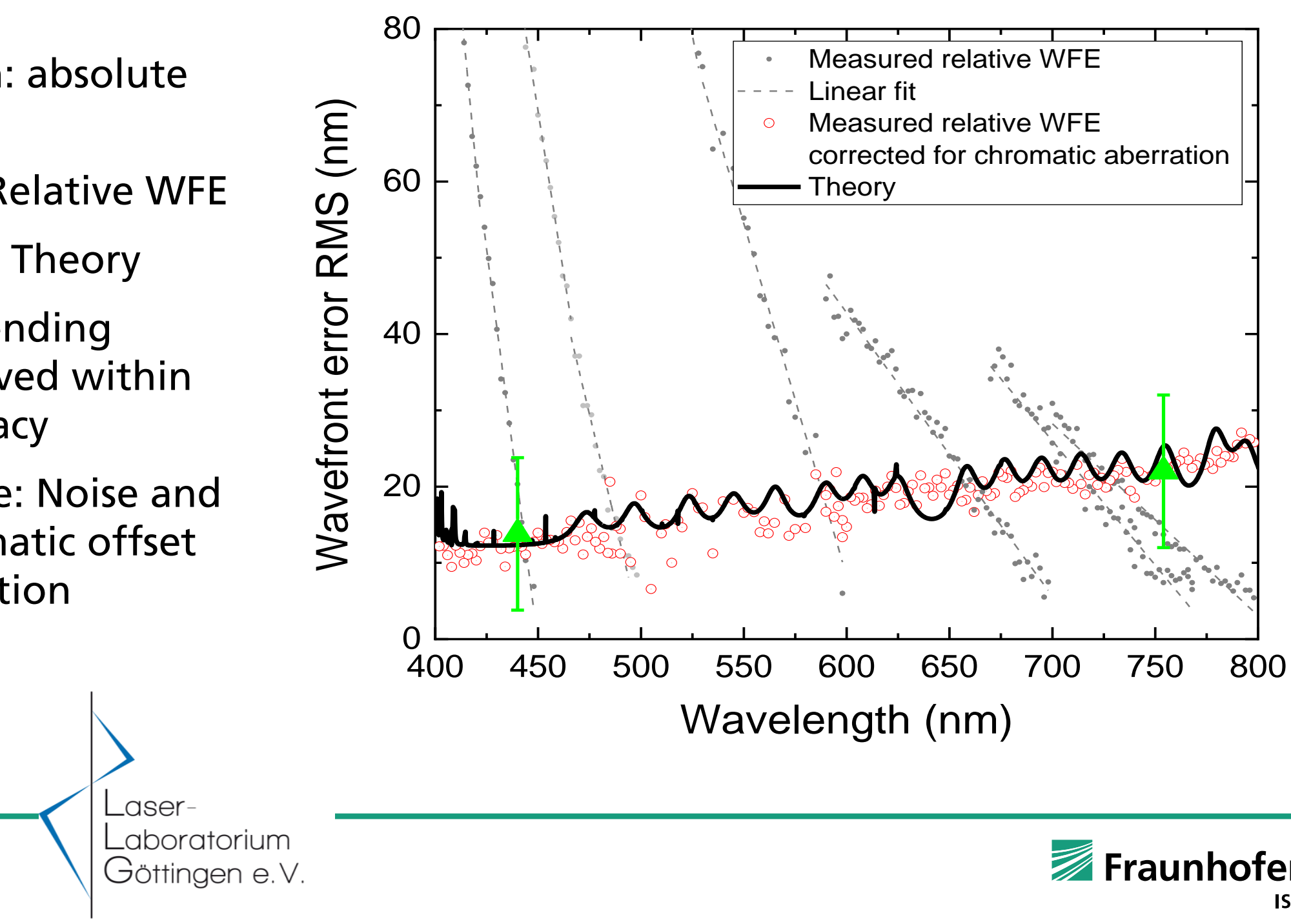


\section{Broadband beam splitter}

\section{Design results if group delay is neglected}

- Black: Optimized design

- Blue and red: Designs if groupd delay

- Can lead to large errors

- More information about design approaches: A. Piegari, Session $14 \mathrm{~b}$

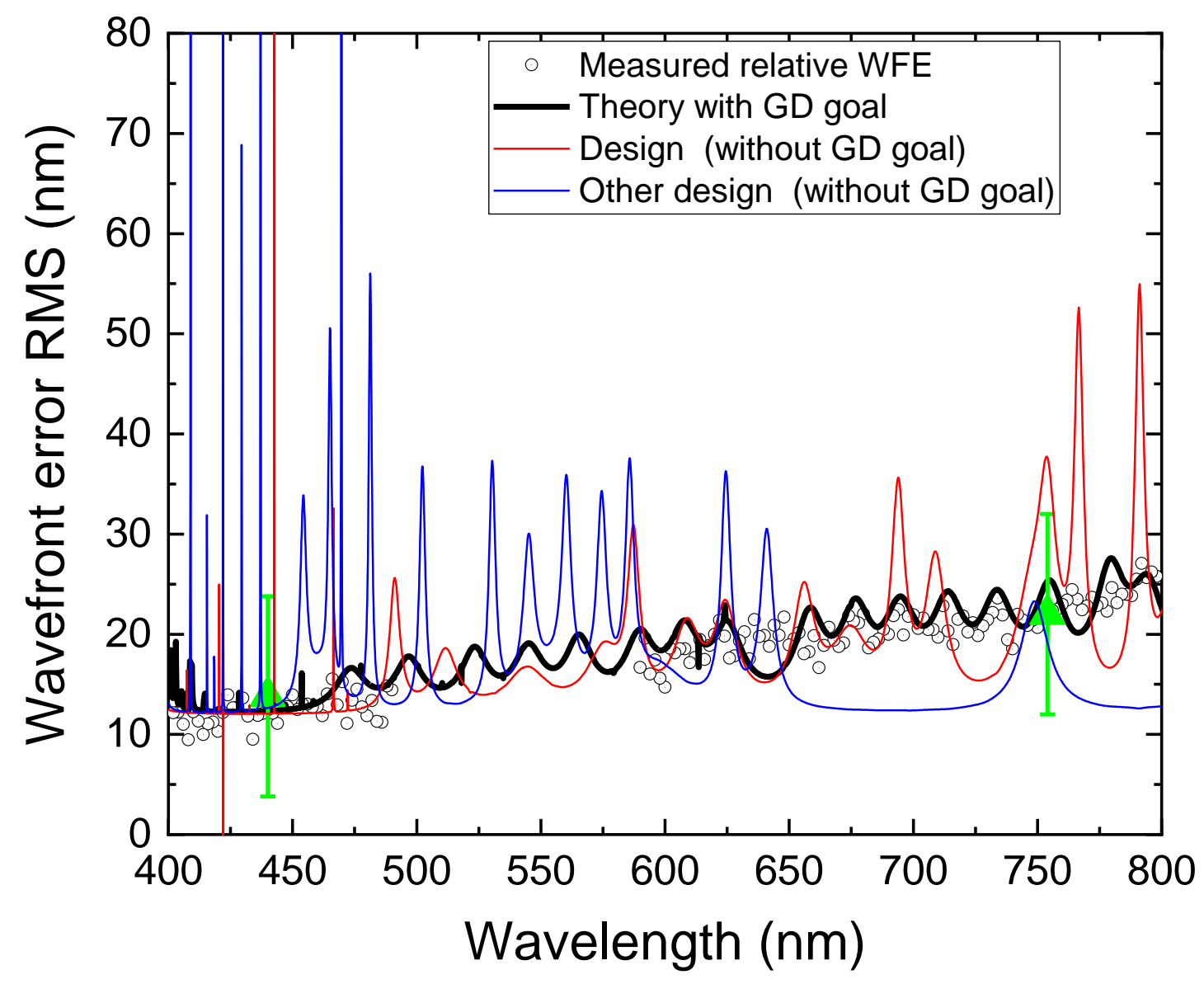




\section{Bandpass coating on convex lens}

Setup

- Spherical lens with coating

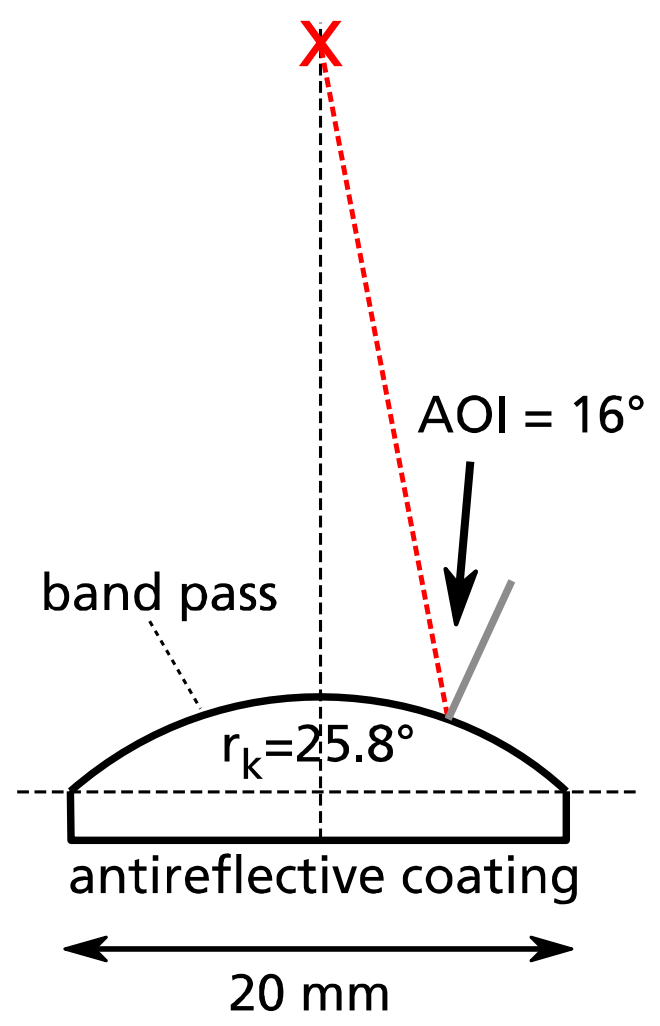

- Band pass filter, $\lambda_{c}=670 \mathrm{~nm}$ without compensation (model)

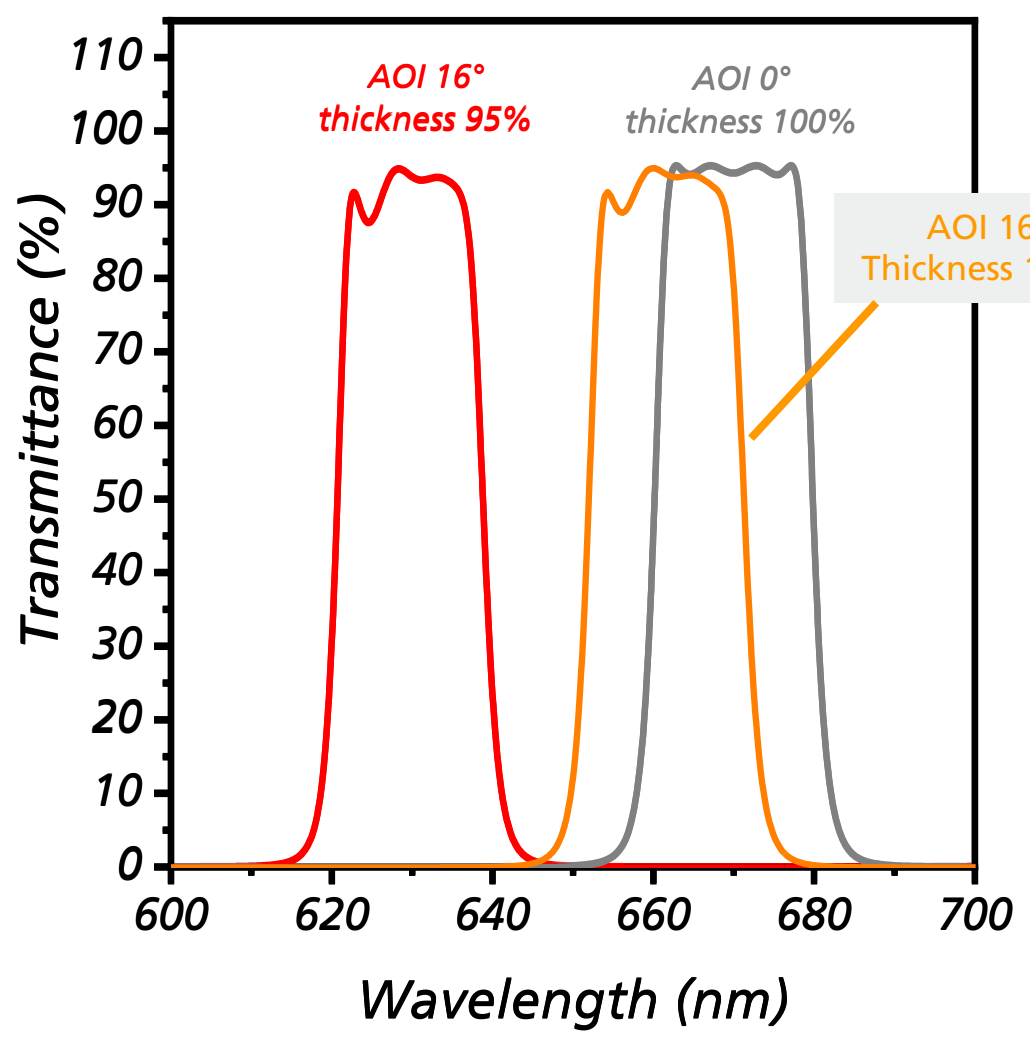




\section{Bandpass coating on convex lens}

\section{Uniformity optimization on flat substrate by simulation}

DSMC model of EOSS compartment

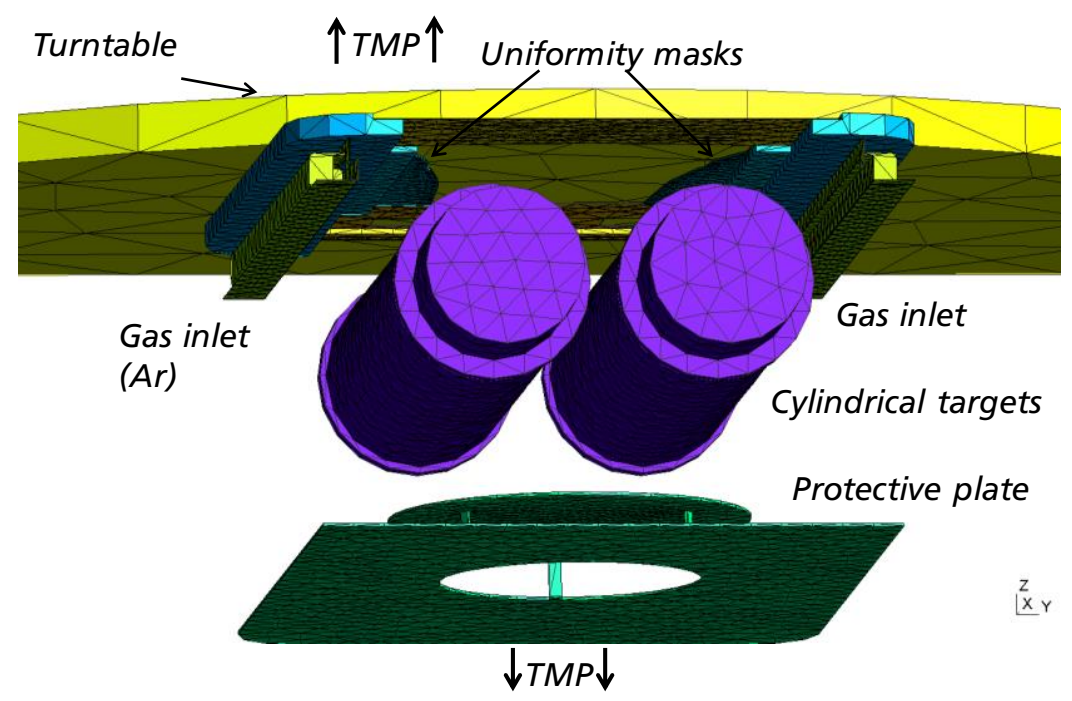

Uniformity on flat substrate: $\pm 0.35 \%$ (nowadays: $\pm 0.2 \%$ )

- DSMC model assumed uniform sputter erosion profile
Achieved uniformity on planar substrate

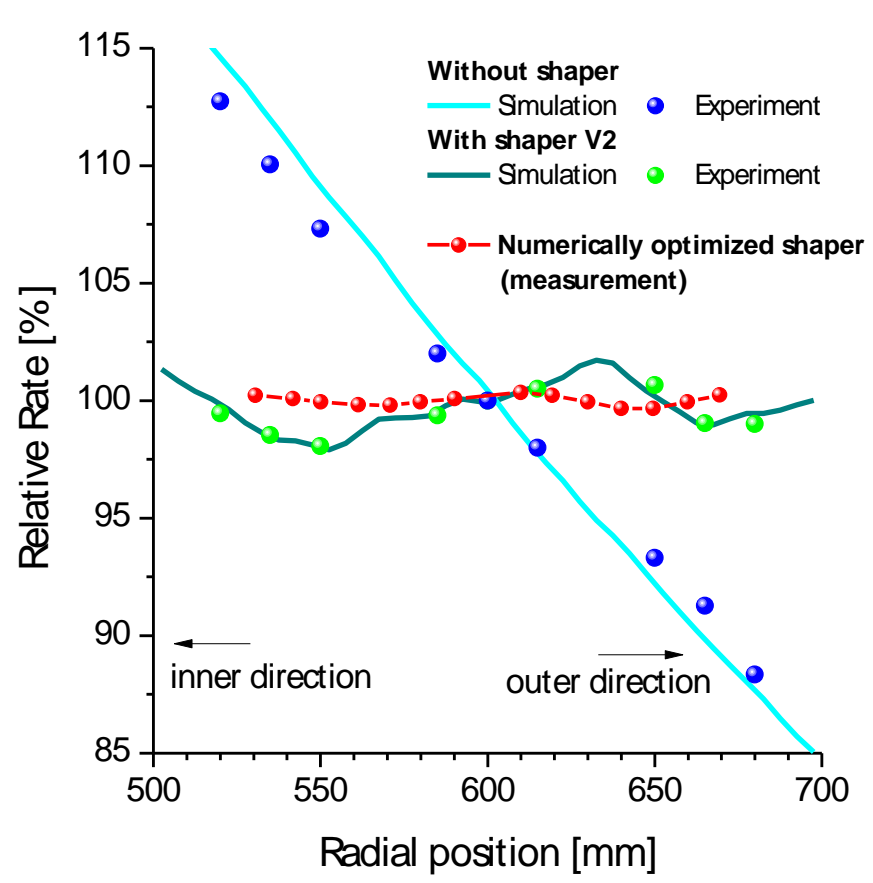

Pflug et al. Surf. Coat. Technol. 241 (2014) 45-9. 


\section{Bandpass coating on convex lens}

New algorithm based on ray tracing \& DSMC

- Partial profiles resulting from individual DSMC runs

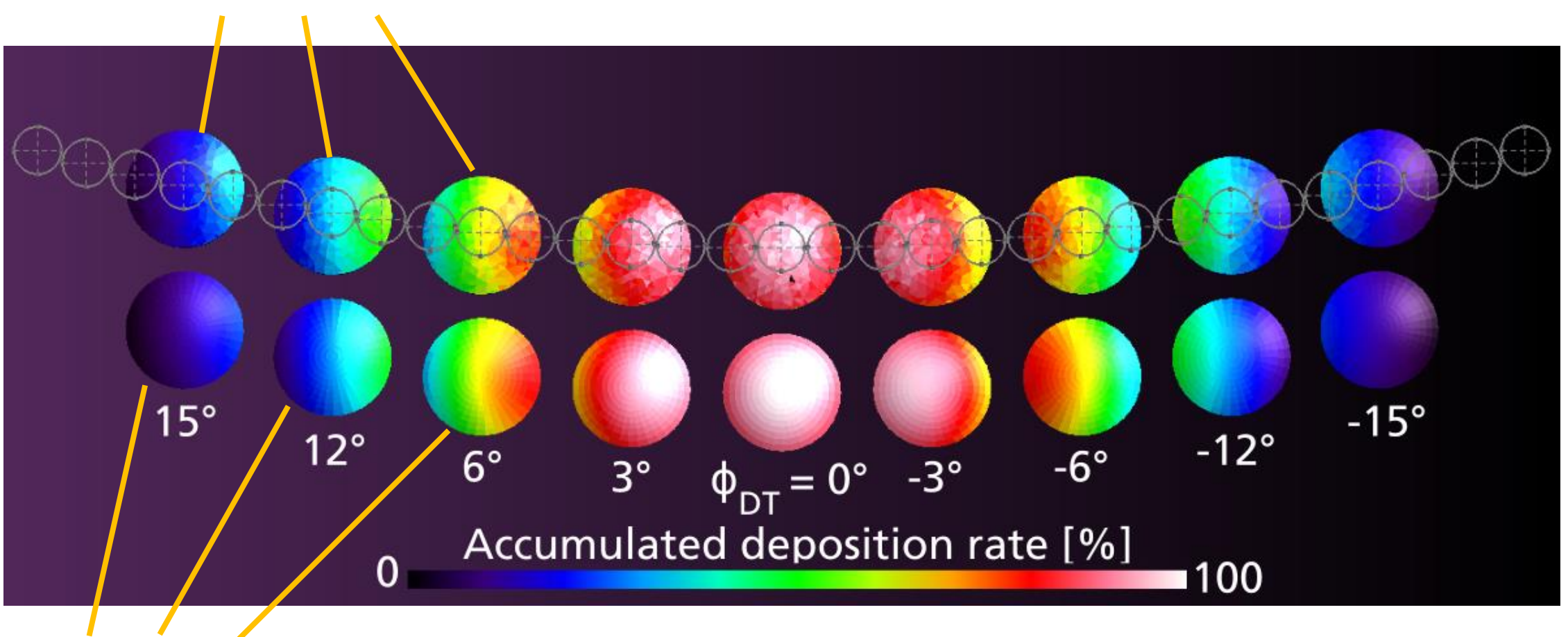

- Partial profiles obtained with projection algorithm

A. Pflug et al, $16^{\text {th }}$ International Conference on Plasma Surface Engineering talk on PSE 2018, to be published in Surf. Coat Technol. (2019) 


\section{Bandpass coating on convex lens}

\section{Tailoring of deposition profile on 3D substrates}

Initial DSMC run

Geometry without masks

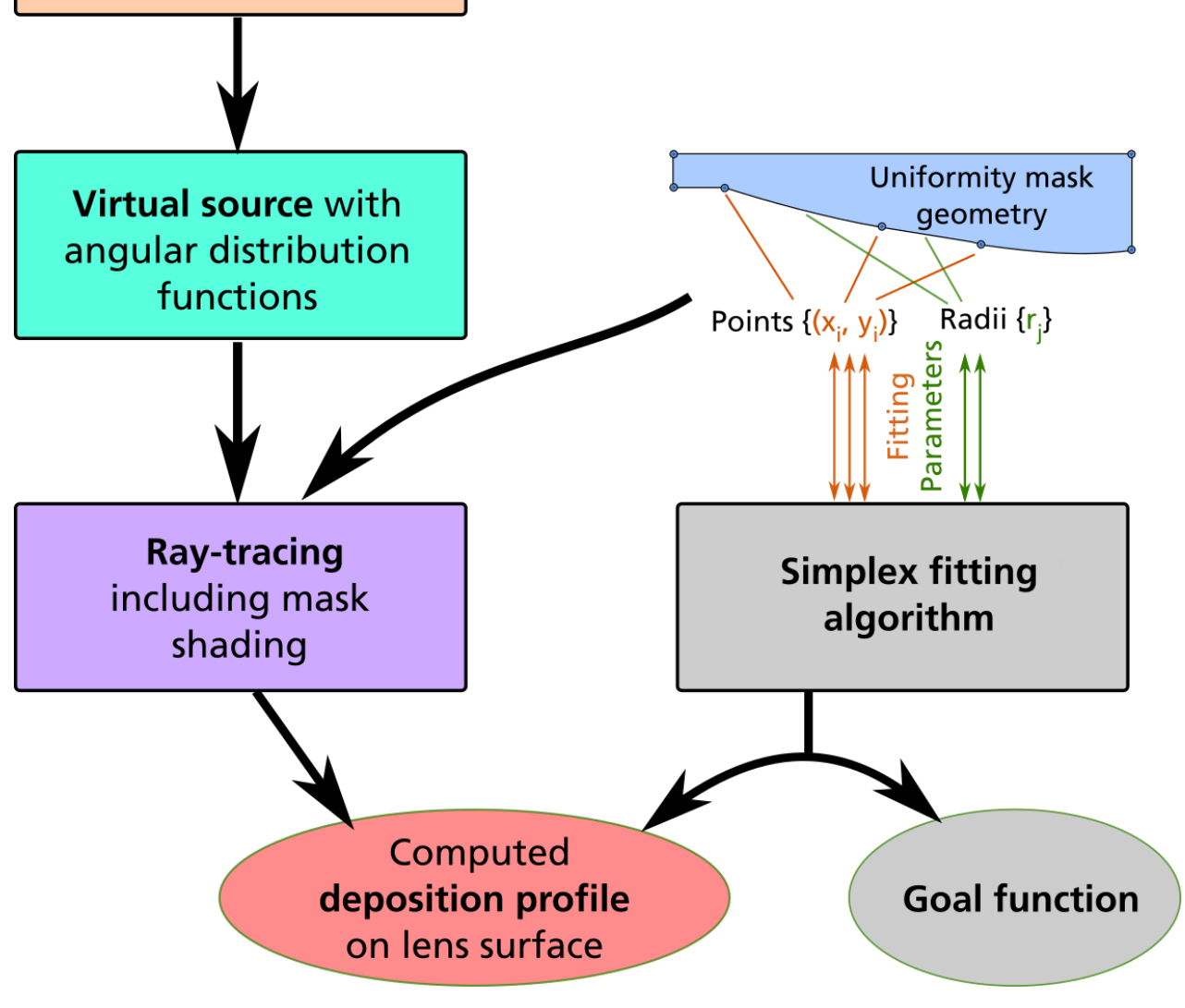

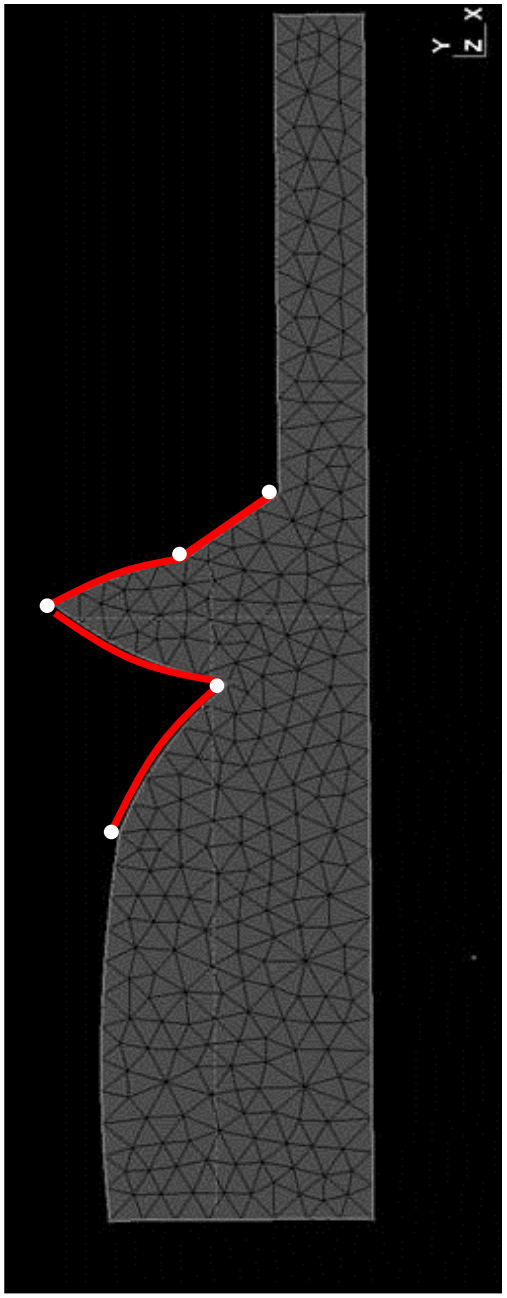




\section{Bandpass coating on convex lens}

"Inverted" deposition profile (simulation)

- Profile without sub rotation

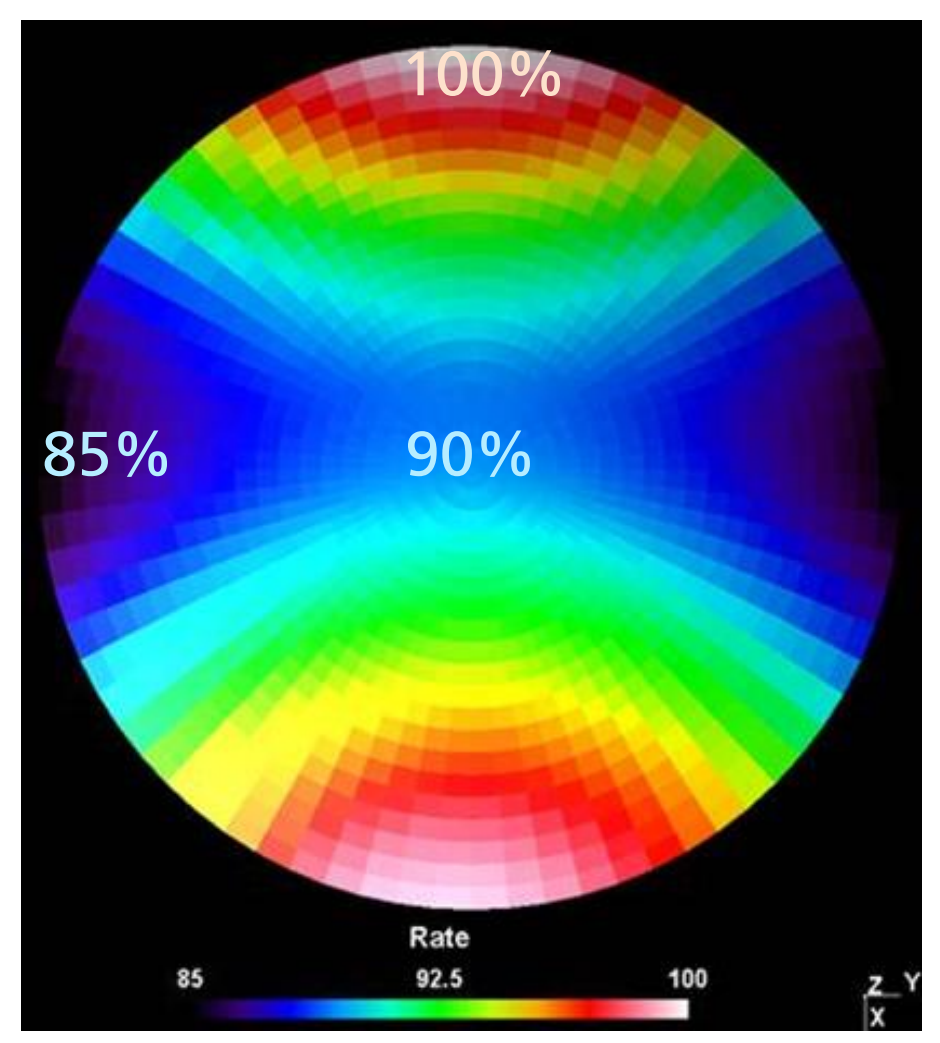

- Profile with sub rotation

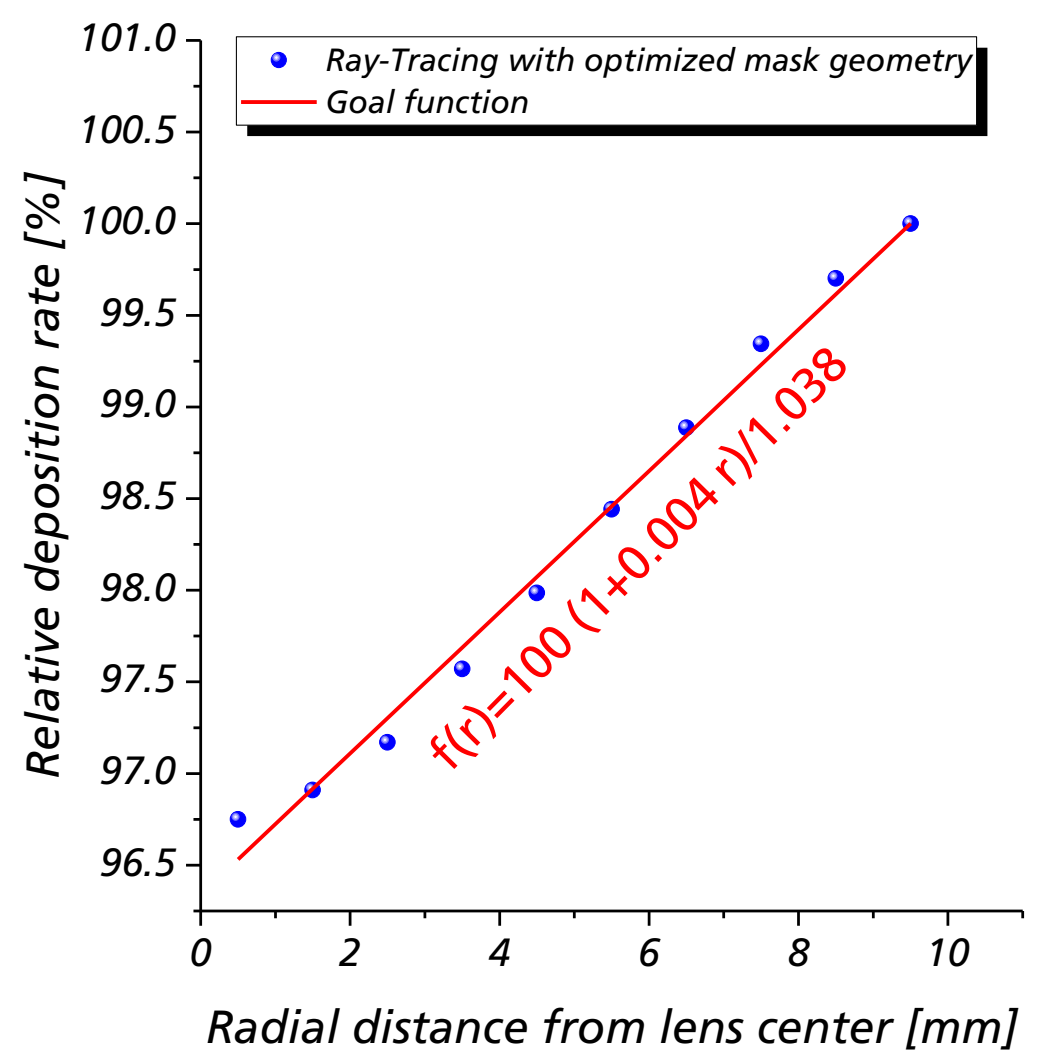




\section{Bandpass coating on convex lens}

\section{"Shadow of the mask" - model vs. experiment}

Very poor agreement of model with experiment

- Reason: Uniform sputter erosion profile assumed

Does not work adequately with sharp masks

= => Determine sputter profiles by PIC-MC 3D plasma simulation

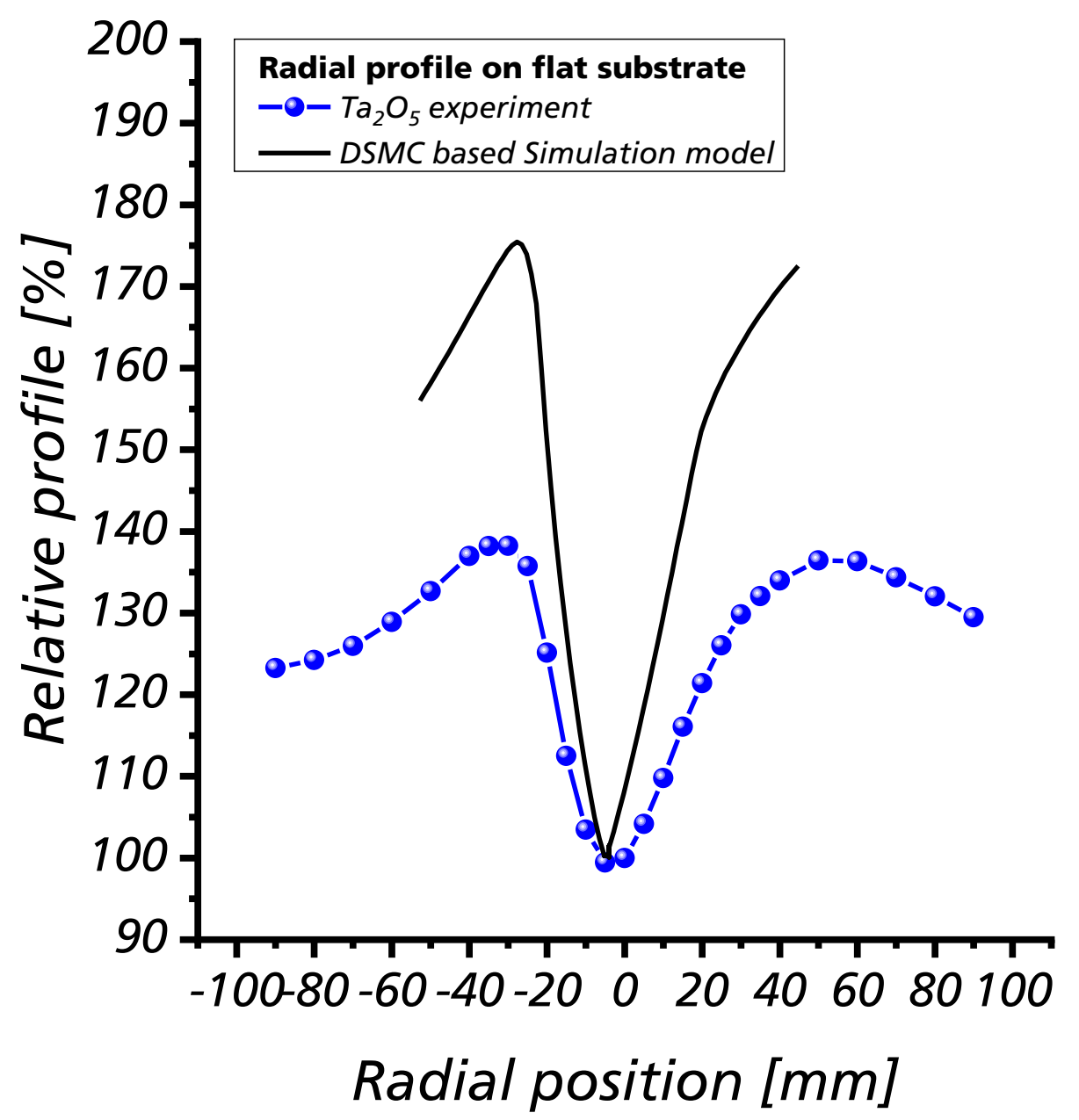




\section{Bandpass coating on convex lens Determination of sputter erosion profile}

Bipolar switching, $40 \mathrm{kHz}$, sin wave, $\mathrm{t}=227.5 \ldots 250 \mu \mathrm{s}$ (10 $10^{\text {th }}$ period)

Averaged $\mathrm{Ar}^{+}$ion flux density

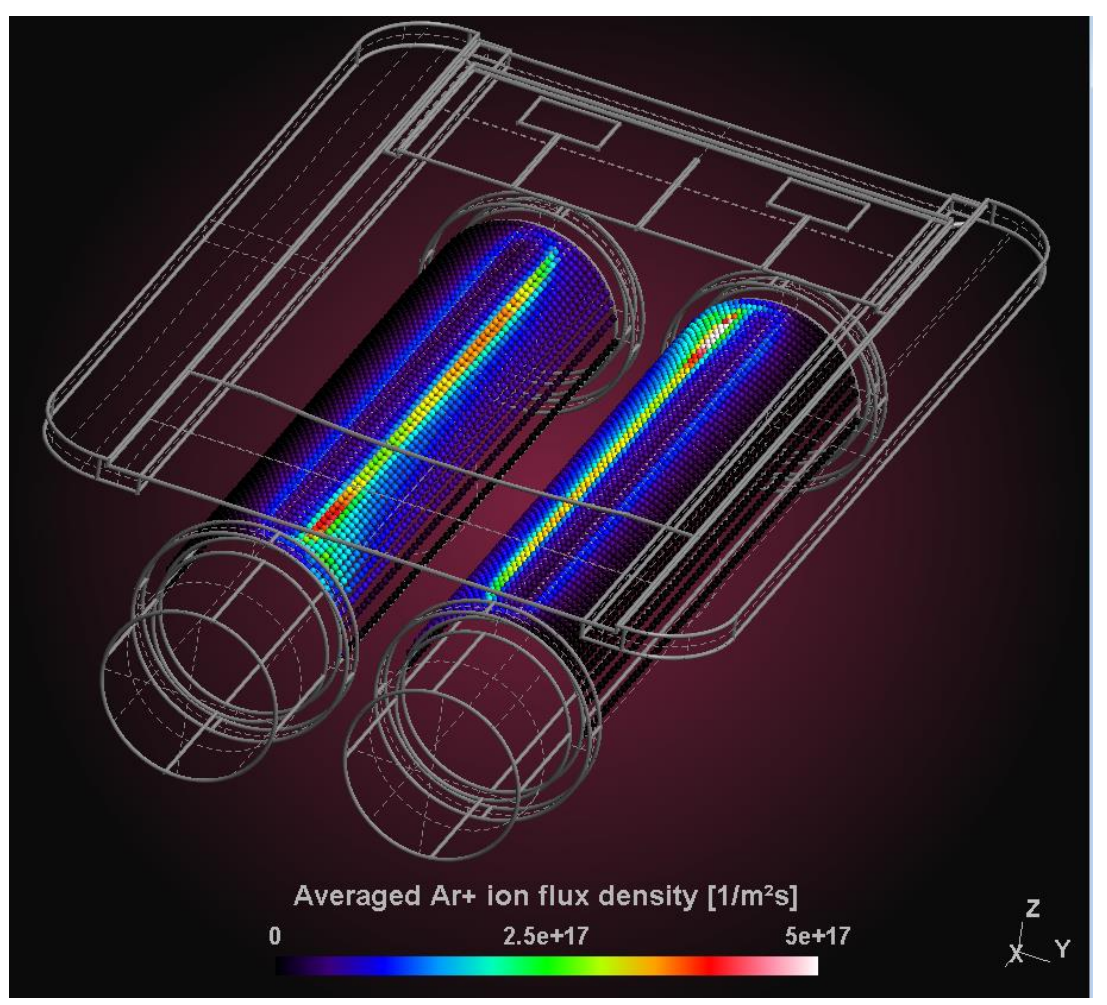

- Ion flux density accumulated over inner and outer racetracks

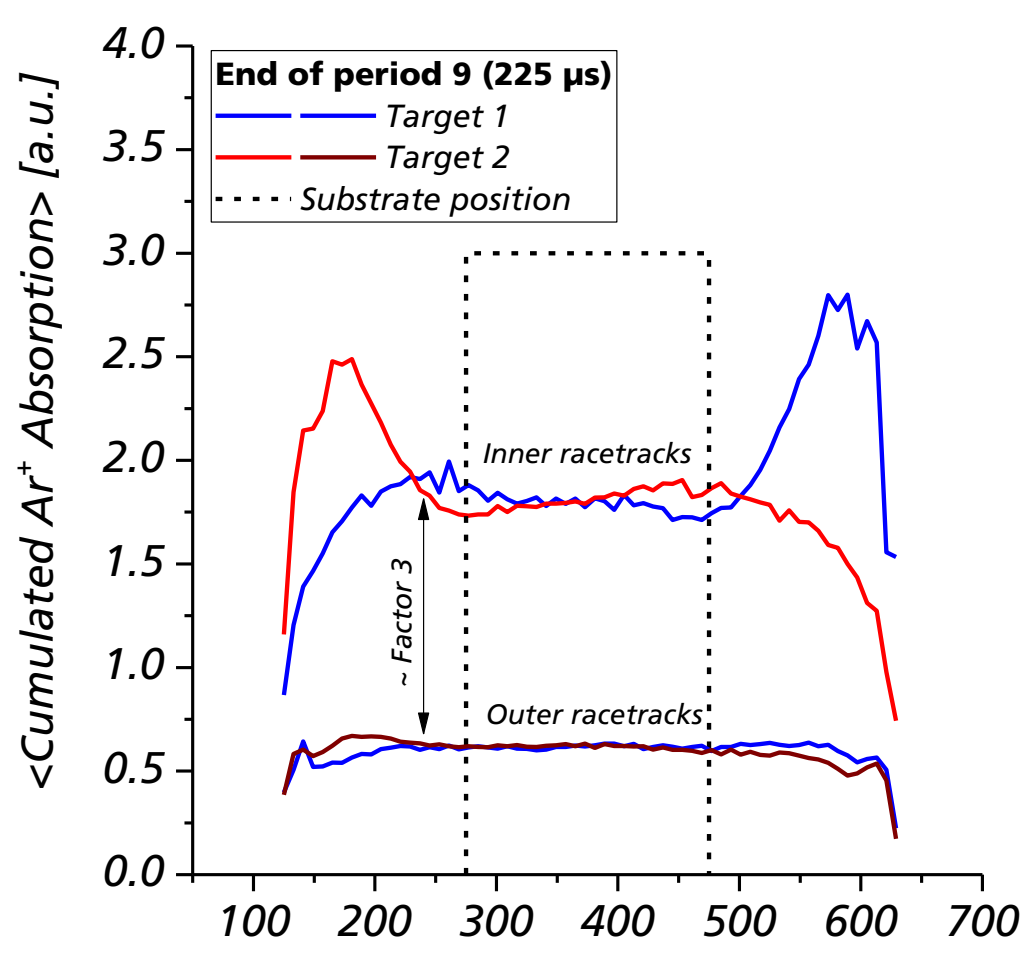

Radial position $X$ [mm] 


\section{Bandpass coating on convex lens \\ Results from improved DSMC transport model}

- Combination of plasma simulation by PIC-MC and subsequent transport simulation yields much better fit with experiment (red curve)

- Just assuming stronger sputter erosion at inner racetracks is not sufficient (pink curve)

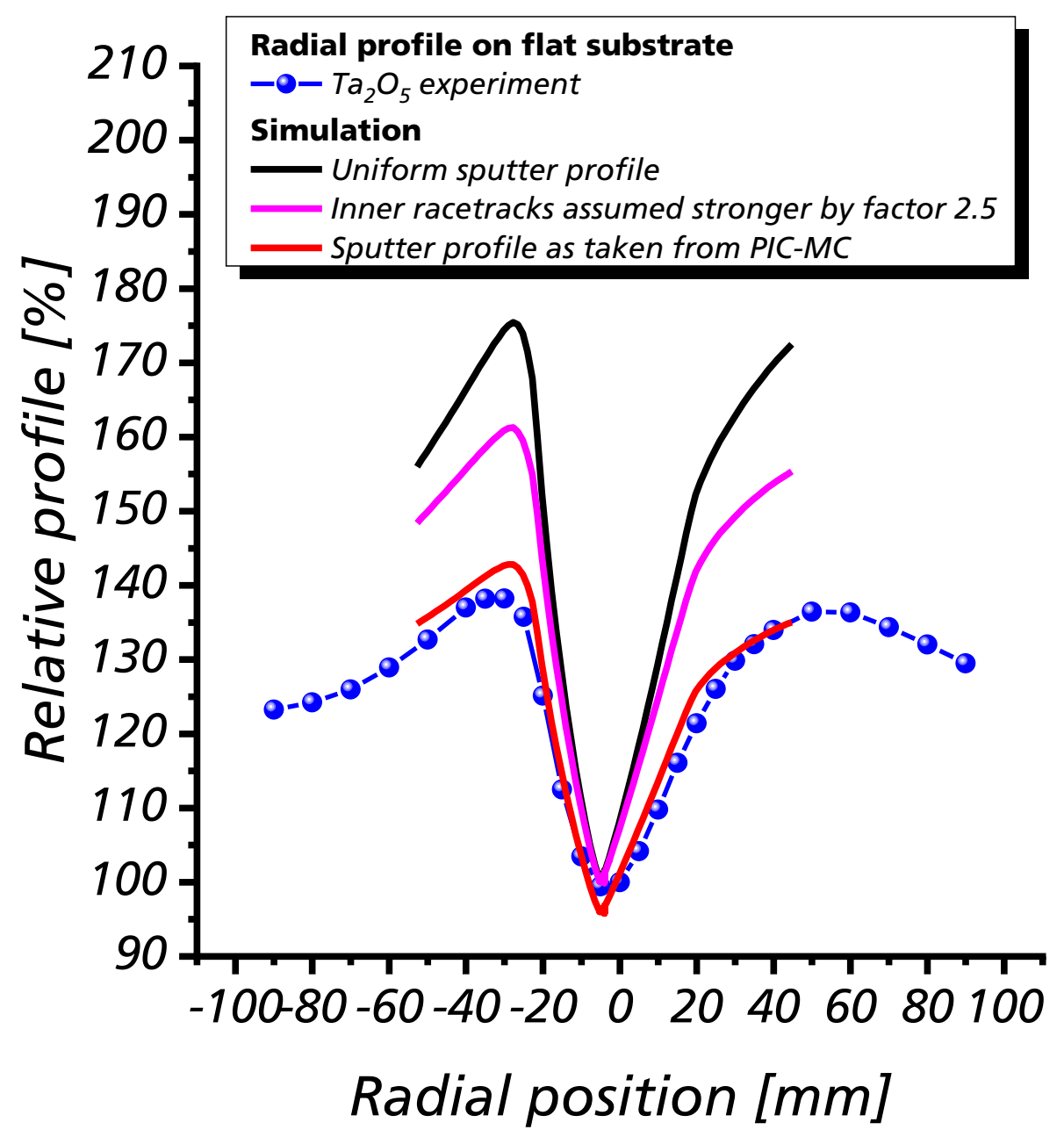




\section{Bandpass coating on convex lens Uniformity mask, $2^{\text {nd }}$ generation}

- Lens and flat substrate with mask

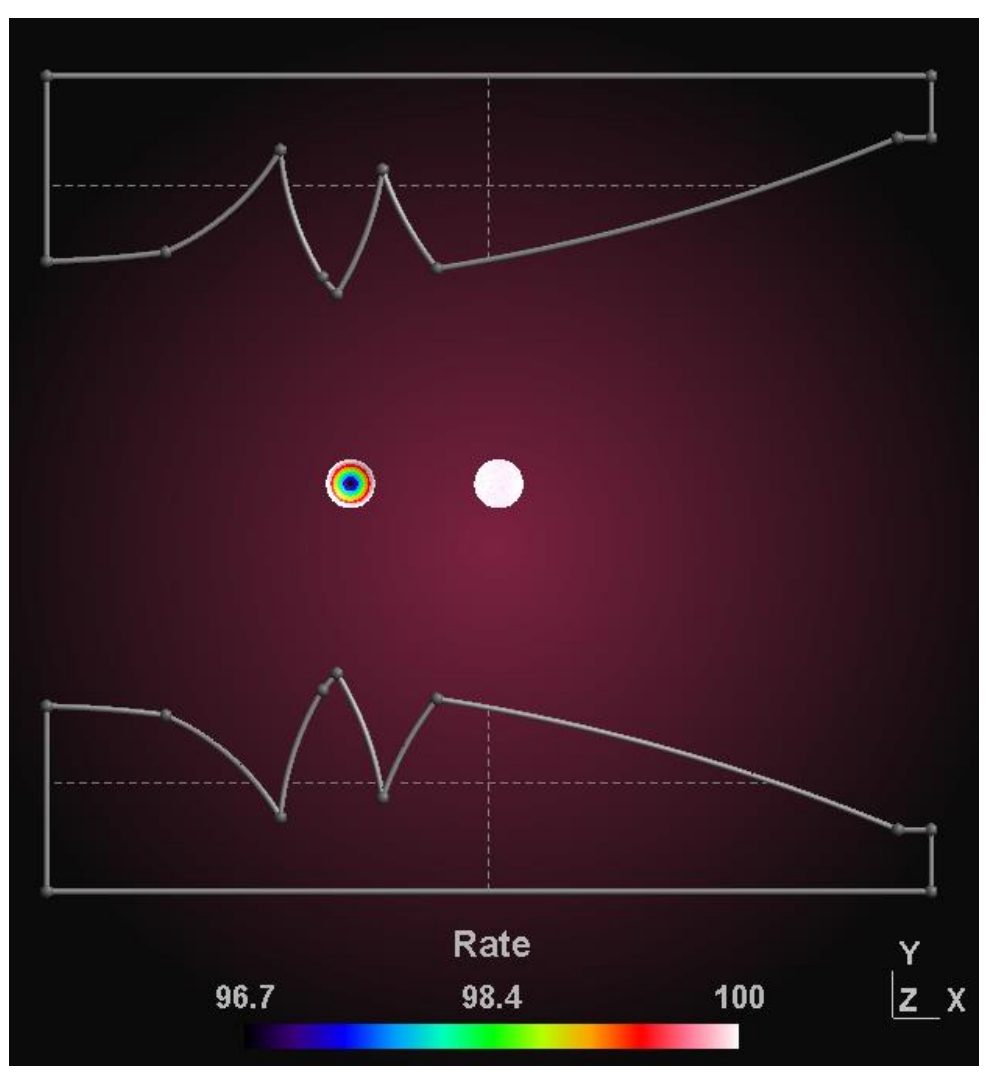

- Computed rate profiles in radial direction

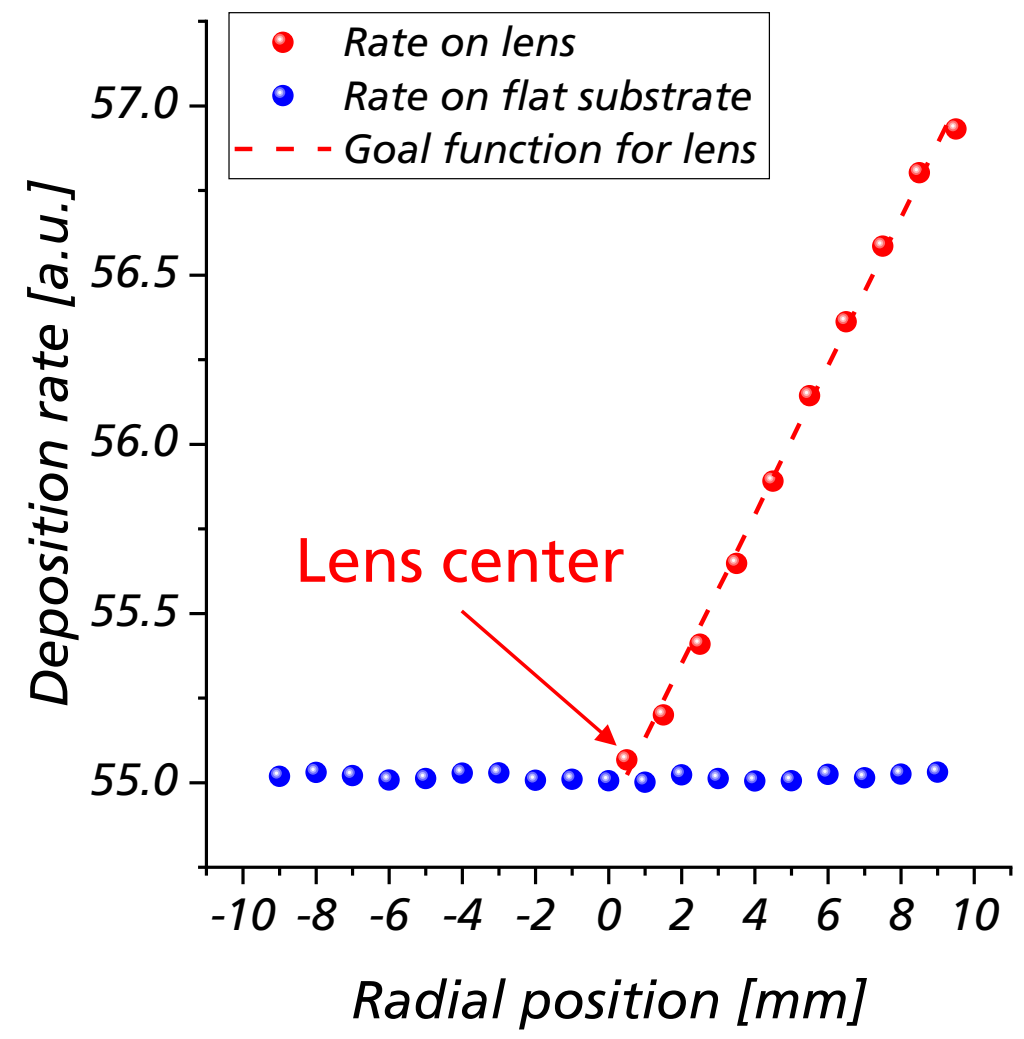




\section{Bandpass coating on convex lens Lens coated with bandpass filter}

- Lens + blocker around bandpass

- $23 \mu \mathrm{m}, 207$ layers

- No backside coating

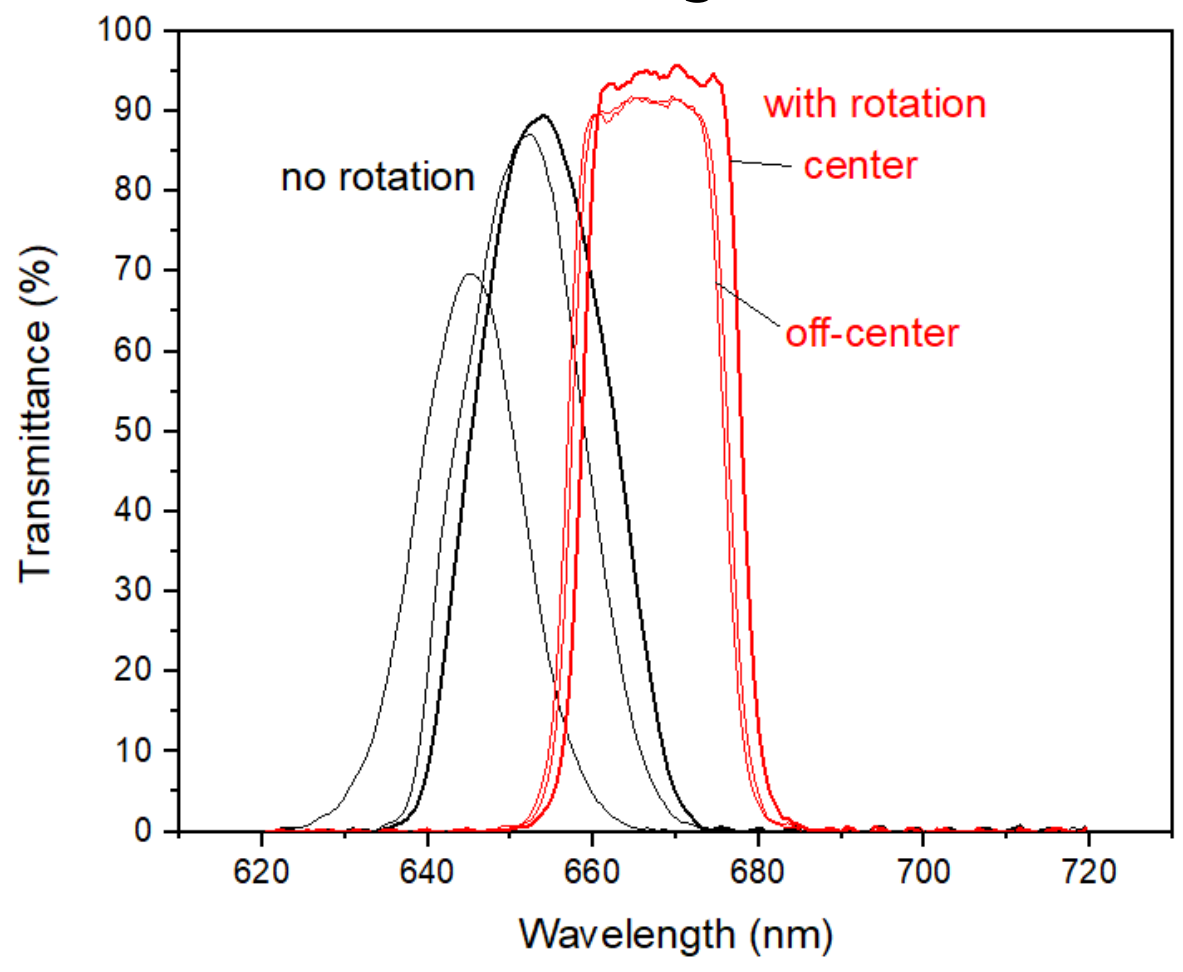

- Blocking

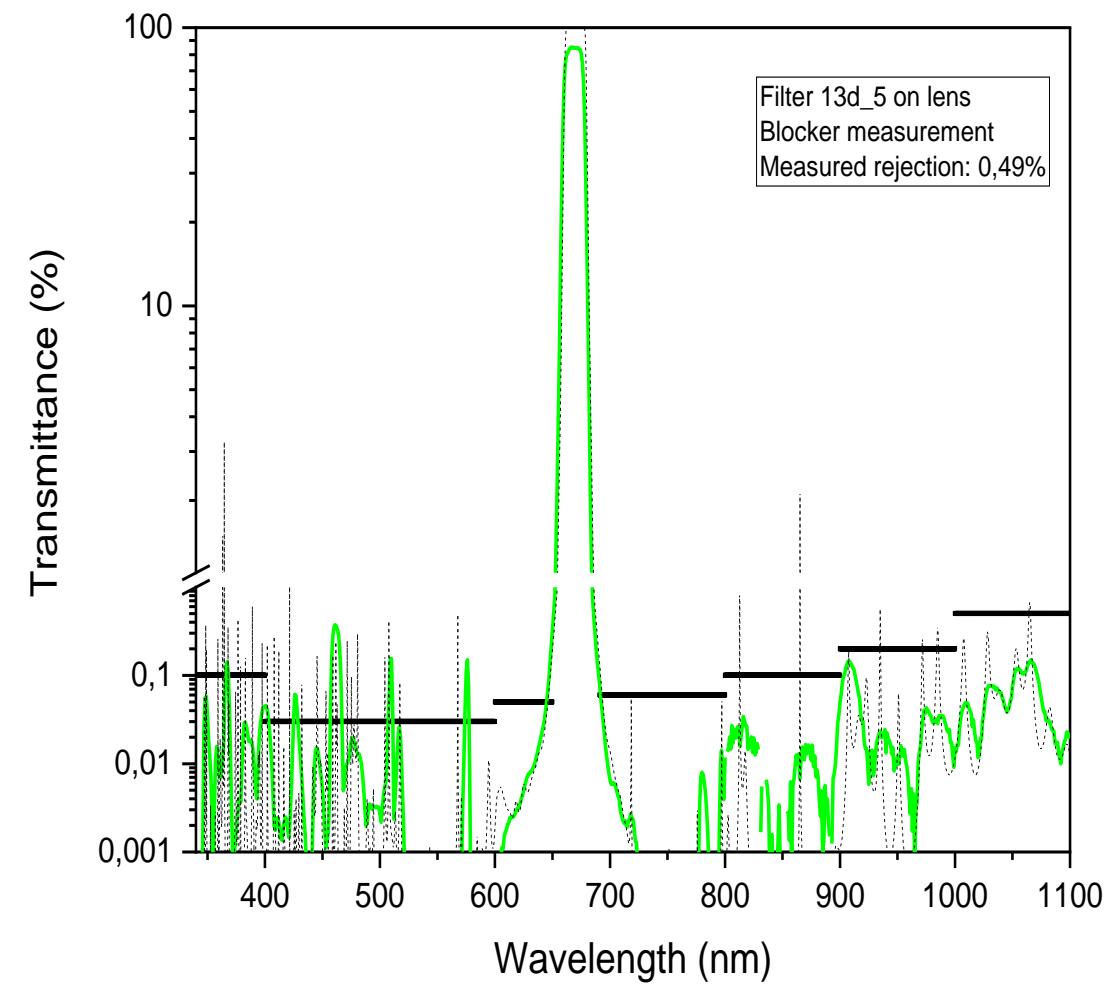




\section{Summary and outlook}

Deposition of broadband beam splitter successful

Significantly reduced wavefront error without resonances

Stable uniformity: $\pm 0.125 \%$

- Measurement of WFE spectra by Hartmann-Shack sensor

- Combination of relative and absolute measurement for fast analysis

- Lens coating: Modelling of the deposition on moving substrates (turntable rotation)

A new fast algorithm to design shaping masks for 3D substrates

- Sputter erosion profile is very important

Mask design by PIC-MC + DSMC simulation only

Realization of coated lenses $\rightarrow$ proof of concept achieved 


\section{Thank you for your attention!}

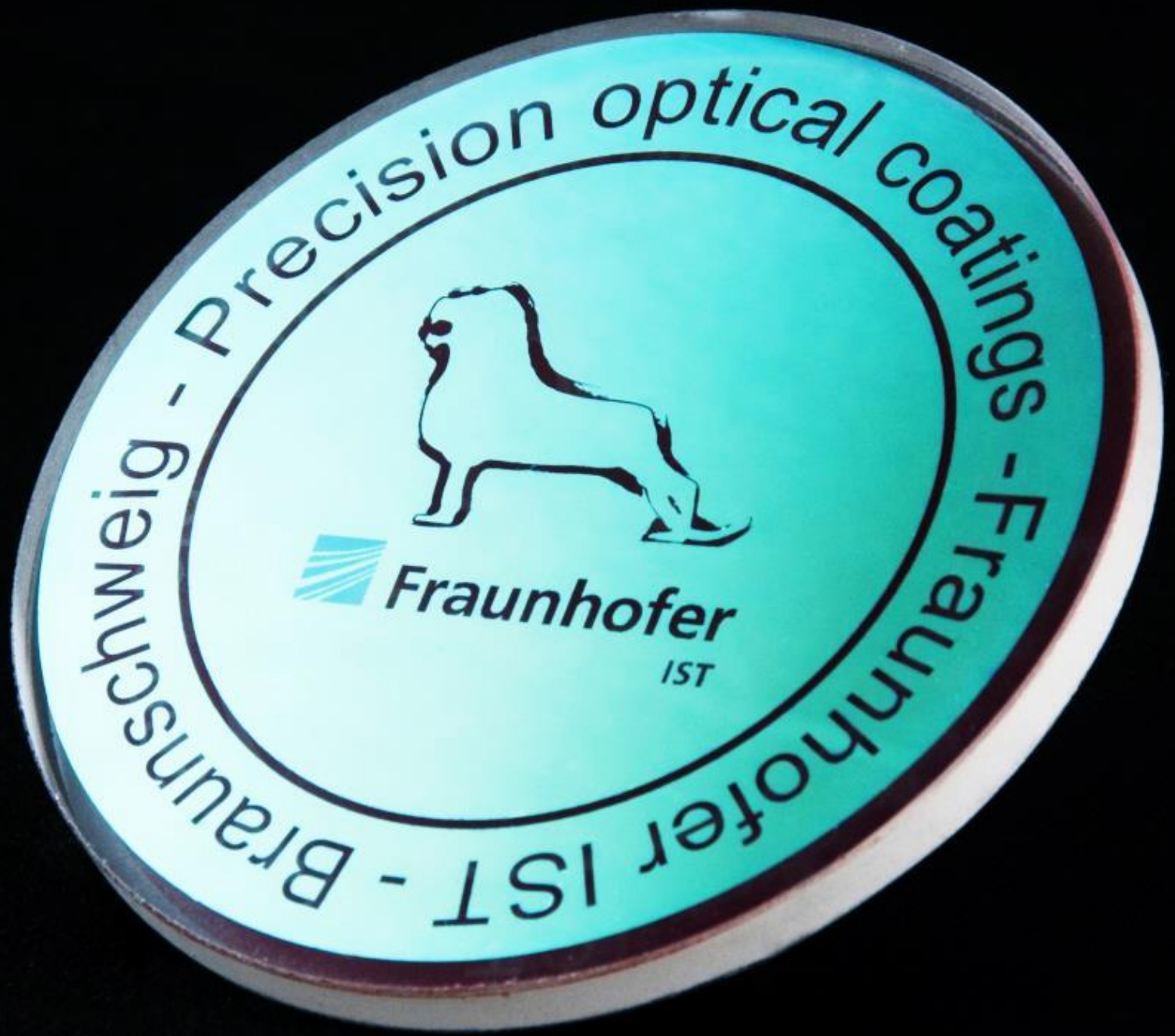

We acknowledge funding of this project by ESA within the activity ITT AO/1-8541/15/NL/PS. 